CARDIOVASCULAR MEDICINE

\title{
Quantification of regional left ventricular function in $Q$ wave and non- $Q$ wave dysfunctional regions by tissue Doppler imaging in patients with ischaemic cardiomyopathy
}

\author{
M Bountioukos, A F L Schinkel, J J Bax, V Rizzello, R Rambaldi, E C Vourvouri, J R T C Roelandt, \\ D Poldermans
}

See end of article for authors' affiliations

Correspondence to: Dr Don Poldermans, Thoraxcentre Room $\mathrm{Ba}$ 300, Department of Cardiology, Erasmus Medical Centre, $\mathrm{Dr}$ Molewaterplein 40, 3015 GD Rotterdam, Netherlands; d.poldermans@ erasmusmc.nl

Accepted 22 May 2003

\begin{abstract}
Objective: To quantify regional left ventricular (LV) function and contractile reserve in $Q$ wave and non-Q wave regions in patients with previous myocardial infarction.

Design: An observational study.

Setting: Tertiary care centre.

Patients: 81 patients with previous myocardial infarction and depressed LV function.

Interventions: All patients underwent surface ECG at rest and pulsed wave tissue Doppler imaging at rest and during low dose dobutamine infusion. The left ventricle was divided into four major regions (anterior, inferoposterior, septal, and lateral). Severely hypokinetic, akinetic, and dyskinetic regions on two dimensional echocardiography at rest were considered dysfunctional.

Main outcome measures: Regional myocardial systolic velocity (Vs) at rest and the change in $V_{s}$ during low dose dobutamine infusion $(\Delta \mathrm{V} s)$ in dysfunctional regions with and without $Q$ waves on surface $E C G$. Results: 220 (69\%) regions were dysfunctional; 60 of these regions corresponded to $Q$ waves and 160 were not related to $Q$ waves. $V s$ and $\Delta V s$ were lower in dysfunctional than in non-dysfunctional regions (mean (SD) Vs $6.2(1.9) \mathrm{cm} / \mathrm{s} \vee 7.1(1.7) \mathrm{cm} / \mathrm{s}(\mathrm{p}<0.001)$, and $\Delta V_{\mathrm{s}} 1.9(1.9) \mathrm{cm} / \mathrm{s} \vee 2.6(2.5) \mathrm{cm} / \mathrm{s}$ $(p=0.009)$, respectively). There were no significant differences in $V_{s}$ and $\Delta V_{s}$ among dysfunctional regions with and without $Q$ waves $\left(Q\right.$ wave regions: $V s 6.2(1.8) \mathrm{cm} / \mathrm{s}, \Delta V_{s} 1.6(2.2) \mathrm{cm} / \mathrm{s}$; non- $Q$ wave regions: Vs $\left.6.3(1.9) \mathrm{cm} / \mathrm{s}, \Delta V_{s} 2.0(2.0) \mathrm{cm} / \mathrm{s}\right)$.

Conclusions: Quantitative pulsed wave tissue Doppler demonstrated that, among dysfunctional regions, $Q$ waves on the ECG do not indicate more severe dysfunction, and myocardial contractile reserve is comparable in $Q$ wave and non- $Q$ wave dysfunctional myocardium.
\end{abstract}

$\mathrm{T}$ raditionally, the classification of myocardial infarctions as transmural or subendocardial has been based on the presence or absence of $\mathrm{Q}$ waves on surface ECG. Even nowadays, chronic pathological Q waves are often considered to represent myocardial regions with more extensive damage than in dysfunctional non-Q wave related regions. However, several studies have shown a poor correlation between $\mathrm{Q}$ waves on the ECG and histopathological findings in patients with a healed myocardial infarction. ${ }^{12}$ Nuclear and echocardiographic techniques used for the detection of viable myocardium have shown that Q wave related regions may contain hibernating myocardium - that is, dysfunctional but viable tissue..$^{3-5}$ Moreover, disappearance of $\mathrm{Q}$ waves and improvement of regional left ventricular (LV) function have been observed after myocardial revascularisation. ${ }^{6-8}$ Quantitative information on regional LV function and contractile reserve in $\mathrm{Q}$ wave and non-Q wave dysfunctional regions is lacking. Accordingly, the aim of this study was to quantify myocardial function at rest and contractile reserve during low dose dobutamine infusion by pulsed wave tissue Doppler imaging (TDI) in Q wave and non-Q wave dysfunctional regions in patients with chronic coronary artery disease and reduced LV function.

\section{METHODS}

Study population

The patient population consisted of 81 consecutive patients with healed Q wave myocardial infarction and ischaemic cardiomyopathy (left ventricular ejection fraction (LVEF) $\leqslant 40 \%$ and symptoms of congestive heart failure). Patients with left bundle branch block, pacemaker rhythm, primary cardiomyopathy or significant valvar disease were not included. All patients underwent resting surface ECG to identify Q wave and non-Q wave regions. Two dimensional echocardiography at rest and during low dose dobutamine infusion ( $10 \mu \mathrm{g} / \mathrm{kg} / \mathrm{min}$ ) was performed to assess regional LV function and myocardial contractile reserve. Pulsed wave TDI at rest and during low dose dobutamine infusion was used to quantify regional wall motion velocity. The LVEF was assessed by radionuclide ventriculography. The hospital medical ethics committee approved the protocol and all patients gave informed consent.

\section{ECG analysis}

Two experienced observers blinded to any other data and using the Selvester QRS screening criteria for Q wave myocardial infarction evaluated the surface ECGs. Accordingly, Q waves were classified as pathological if the following criteria were met: (1) Q wave $\geqslant 30 \mathrm{~ms}$ in aVF; (2) $\mathrm{Q}$ wave $\geqslant 40 \mathrm{~ms}$ in I and aVL; (3) Q wave $\geqslant 40 \mathrm{~ms}$ in two or more of V4-V6; (4) R wave $\geqslant 40 \mathrm{~ms}$ in $\mathrm{Vl}$; (5) any Q wave in $\mathrm{V} 2$; and $(6) \mathrm{R}$ wave $\leqslant 0.1 \mathrm{mV}$ and $\leqslant 10 \mathrm{~ms}$ in lead $\mathrm{V} 2 .{ }^{9}{ }^{10}$ Pathological Q waves were assigned to four LV regions: anterior (preserved R wave in VI and pathological Q wave in

Abbreviations: LV, left ventricular; LVEF, left ventricular ejection fraction; TDI, tissue Doppler imaging; Vs, systolic ejection velocity 
one or more of leads V2-V5); septal (pathological Q waves in V1, V2, or V3); lateral (pathological Q wave in more than one of leads I, aVL, or V6); or inferoposterior (pathological Q wave in more than one of leads II, III, aVF). ${ }^{4}$

\section{Two dimensional echocardiography}

A commercially available imaging system equipped with a 1.8 MHz transducer and second harmonic imaging to optimise endocardial border visualisation was used to record two dimensional echocardiograms (Hewlett Packard Sonos 5500, Andover, Massachusetts, USA). Four standard views were recorded and two experienced reviewers visually scored the digitised echocardiograms. Regional wall motion and systolic wall thickening were scored using a 16 segment model and a five point grading scale: 1 is normal, 2 is mildly hypokinetic, 3 is severely hypokinetic, 4 is akinetic, 5 is dyskinetic. The 16 segment model was converted to correspond to the four major regions (anterior, inferoposterior, septal, and lateral). A region was considered dysfunctional if one or more segments in the region were severely hypokinetic, akinetic, or dyskinetic on two dimensional echocardiography.

\section{Tissue Doppler imaging}

Pulsed wave TDI was performed with the same system used for the assessment of wall motion abnormalities, with a pulse repetition frequency of $45-60 \mathrm{KHz}$. A sample volume of $4 \mathrm{~mm}^{3}$ was used. Myocardial velocity was sampled in apical views (posterior septum, anterior septum, and lateral, inferior, anterior, and posterior walls) close to the mitral annulus and during a minimum of five consecutive beats to minimise the variability induced by respiration. The depth of the sample volume of every wall was kept constant during dobutamine stress echocardiography to make sure that LV myocardium was sampled close to the mitral annulus.

The Doppler velocity profiles and ECG tracings were simultaneously stored on optical disk. All the measurements were performed off line with a computer assisted drawing system. The velocity values $(\mathrm{cm} / \mathrm{s})$ were obtained on calibrated still frames by manually measuring the distance between the zero baselines and the peak Doppler profile of the ejection phase in reference to the ECG. Cardiac cycles with extrasystolic or post-extrasystolic beats or rhythm disturbance were excluded. Recordings and measurements were made at baseline and at low dose $(10 \mu \mathrm{g} / \mathrm{kg} / \mathrm{min})$ dobutamine infusion. The myocardial walls were related to the four major LV regions (anterior, septal, lateral, inferoposterior) by calculating the mean Doppler velocities of the anterior and posterior septum (septal region) and of the inferior and posterior wall (inferoposterior region). As described previously, a cut off value of $\geqslant 1 \mathrm{~cm} / \mathrm{s}$ in systolic ejection velocity (Vs) at low dose dobutamine infusion was used to define viability of dysfunctional regions. ${ }^{11}$

\section{Radionuclide ventriculography}

To assess the LVEF, radionuclide ventriculography at rest was performed in all patients. A small field of view gamma camera system (Orbiter, Siemens, Erlangen, Germany) was used oriented in a $45^{\circ}$ left anterior oblique position with a 5$10^{\circ}$ caudal tilt. After injection of Tc-99m (740 MBq), radionuclide ventriculography was performed at rest with the patient in supine position. The LVEF was calculated by standard methods (Odyssey VP, Picker, Cleveland, Ohio, USA).

\section{Statistical analysis}

All continuous data are expressed as mean (SD) and percentages are rounded. Continuous variables were compared using Student's $t$ test for unpaired samples. Differences between proportions were compared using the $\chi^{2}$ test. A value of $\mathrm{p}<0.05$ was considered significant.

\section{RESULTS}

Study patients had a mean age of 61 (10) years (range 43-78 years). Mean LVEF was 32 (9)\% (range 10-40\%). Table 1 shows the baseline patient characteristics.

\section{ECG results}

An abnormal Q wave pattern was present in 89 regions; 27 $(30 \%)$ of them were anterior, 27 (30\%) inferoposterior, 19 $(22 \%)$ septal, and 16 (18\%) lateral regions. On average, the patients had $1.1(0.5)$ regions associated with a $\mathrm{Q}$ wave pattern on the ECG.

\section{Wall motion analysis}

A total of 320 of 324 (99\%) regions were analysed both by two dimensional echocardiography and by pulsed wave TDI. Assessment of myocardial wall motion by two dimensional echocardiography showed that 220 (69\%) regions were dysfunctional (severely hypokinetic, akinetic, or dyskinetic). Among them, 60 (27\%) regions were Q wave related: 16 (27\%) anterior, 17 (28\%) inferoposterior, 13 (22\%) septal, and $14(23 \%)$ lateral regions. Figure 1 illustrates the distribution of myocardial regions.

\section{Tissue Doppler imaging}

The mean Vs of dysfunctional regions was $6.2(1.9) \mathrm{cm} / \mathrm{s}$ and of non-dysfunctional regions was $7.1(1.7) \mathrm{cm} / \mathrm{s}(\mathrm{p}<0.001)$. Figure 2 presents the mean systolic TDI velocities at baseline and at low dose dobutamine infusion. Vs increased significantly at low dose dobutamine infusion in both Q wave and non-Q wave dysfunctional regions ( $\mathrm{Q}$ wave regions: from $6.2(1.8) \mathrm{cm} / \mathrm{s}$ at rest to $7.8(2.9) \mathrm{cm} / \mathrm{s}$ at low dose $(\mathrm{p}<0.001)$; non-Q wave regions: from $6.3(1.9) \mathrm{cm} / \mathrm{s}$ at rest to $8.3(2.8) \mathrm{cm} / \mathrm{s}$ a low dose; $\mathrm{p}<0.001)$. There was no significant difference in Vs at rest and at low dose dobutamine between $\mathrm{Q}$ wave related and non-Q wave related dysfunctional regions (baseline 6.2 (1.8) cm/s $v 6.3$ (1.9) cm/s; $\mathrm{p}=0.948$; low dose dobutamine infusion $7.8(2.9) \mathrm{cm} / \mathrm{s} v$ $8.3(2.8) \mathrm{cm} / \mathrm{s} ; \mathrm{p}=0.268$ in $\mathrm{Q}$ wave and non-Q wave regions, respectively).

The increase of Vs from baseline to low dose dobutamine infusion $(\Delta V s)$ was higher in non-dysfunctional regions $(2.6$ $(2.5) \mathrm{cm} / \mathrm{s} v 1.9 \quad(1.9) \mathrm{cm} / \mathrm{s}$ in dysfunctional regions; $\mathrm{p}=0.009)$. No difference in $\Delta \mathrm{Vs}$ was found between $\mathrm{Q}$

\begin{tabular}{ll} 
Table 1 & Baseline clinical characteristics \\
\hline Male sex & $69(85 \%)$ \\
Age (years) & $61(10)$ \\
LVEF (\%) & $323(10) 8$ \\
Diabetes mellitus* & $6(7 \%)$ \\
Hypercholesterolaemiat & $36(44 \%)$ \\
Systemic hypertensionf & $28(35 \%)$ \\
Current smoking & $30(37 \%)$ \\
History of angina & $50(62 \%)$ \\
Previous revascularisation & $13(16 \%)$ \\
PTCA & $20(35 \%)$ \\
CABG & \\
Medication & $40(49 \%)$ \\
B Blockers & $51(63 \%)$ \\
ACE inhibitors &
\end{tabular}

Data are presented as number (\%) of patients or mean (SD). *Patients receiving oral antidiabetic agents or insulin. †Defined as a total cholesterol $\geqslant 6.4 \mathrm{mmol} / \mathrm{l}$ or treatment with lipid lowering medication.

$\neq$ Defined as blood pressure $\geqslant 140 / 90 \mathrm{~mm} \mathrm{Hg}$ or treatment with antihypertensive medication.

$\mathrm{ACE}$, angiotensin converting enzyme; $\mathrm{CABG}$, coronary artery bypass grafting; LVEF, left ventricular ejection fraction; PTCA, percutaneous transluminal coronary angioplasty. 


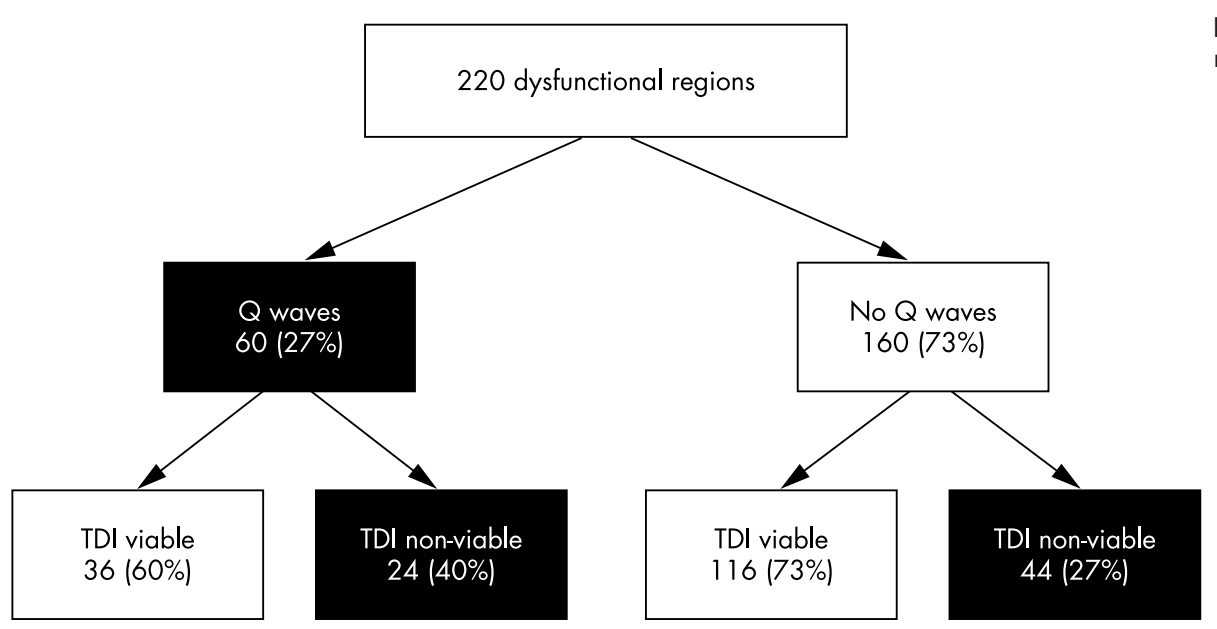

Figure 1 Distribution of myocardial regions in study patients.

wave and non-Q wave related regions $(1.6(2.2) \mathrm{cm} / \mathrm{s} v 2.0$ $(2.0) \mathrm{cm} / \mathrm{s}$, respectively; $\mathrm{p}=0.129)$. Table 2 shows the TDI velocities found in each of the four myocardial regions. Considering an increase in baseline Vs of $\geqslant 1 \mathrm{~cm} / \mathrm{s}$ at low dose dobutamine infusion as the cut off value to define viability, $60 \%$ and $73 \%$ of Q wave and non-Q wave dysfunctional regions, respectively, were viable ( $p=0.104)$ (fig 1 ).

\section{DISCUSSION}

In the present study, pulsed wave TDI, a relatively new echocardiographic technique with high temporal resolution for the quantification of myocardial wall motion velocity, was used. The results show that Q wave regions on surface ECG do not necessarily indicate more advanced myocardial dysfunction than do dysfunctional non-Q wave regions. Although Vs was found to be significantly higher in nondysfunctional regions, the comparison between dysfunctional $\mathrm{Q}$ wave and dysfunctional non-Q wave related regions showed no difference, both at rest and at low dose dobutamine infusion. Furthermore, when a cut off value of $\Delta V s \geqslant 1 \mathrm{~cm} / \mathrm{s}$ from rest to low dose dobutamine infusion was used to discriminate viable from non-viable dysfunctional regions, the prevalence of myocardial viability was similar in regions with and without $\mathrm{Q}$ waves.

\section{Comparison with previous studies}

The detection of viable tissue in patients with ischaemic cardiomyopathy is considered a critical issue affecting patients' quality of life and survival. The decision for

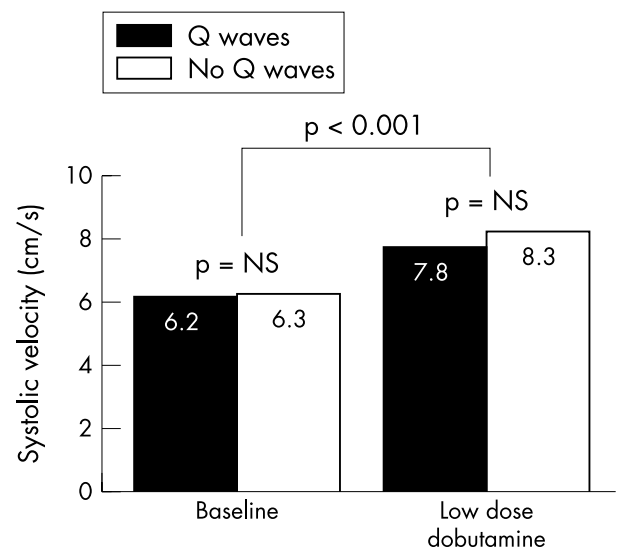

Figure 2 Myocardial systolic velocities in $Q$ wave and non- $Q$ wave dysfunctional regions. revascularisation is influenced by the presence or absence of viability. Several studies have reported the beneficial effect of revascularisation on LV function and on survival in patients with LV dysfunction and a substantial amount of viable myocardium. ${ }^{7812-14}$ Various non-invasive techniques (mainly nuclear and echocardiographic) have been used to assess myocardial viability in patients with LV dysfunction caused by chronic coronary artery disease. For many years, Q waves in dysfunctional regions were believed to reflect irreversibly scarred myocardial tissue and Q waves were associated with more severely damaged myocardium. However, there is some evidence from histopathological studies that residual viable tissue may be present in dysfunctional regions with Q waves on the ECG. Moreover, non-invasive imaging techniques have shown signs of viable myocardium in Q wave infarcted regions. The proportion of viable tissue found in patients with $\mathrm{Q}$ wave myocardial infarction varies between studies that used positron emission tomography to assess viability, ranging from $36-68 \%$. $^{3-5}$

$\mathrm{Q}$ waves represent the initial $40 \mathrm{~ms}$ of depolarisation. When a region is depolarised after this time period, as is the case for basal myocardial regions, Q waves are not produced on ECG. In addition, there are many Q wave equivalent ECG signs that can be missed and be considered to be a lack of

Table 2 Pulsed wave myocardial systolic velocities of $Q$ wave and non- $Q$ wave dysfunctional regions at rest and during low dose dobutamine infusion

\begin{tabular}{|c|c|c|c|}
\hline Region & $Q$ wave regions & $\begin{array}{l}\text { Non- } Q \text { wave } \\
\text { regions }\end{array}$ & p Value \\
\hline \multicolumn{4}{|c|}{ Systolic velocity at rest $(\mathrm{cm} / \mathrm{s})$} \\
\hline Anterior & $6.6(1.9)$ & $6.2(2.0)$ & NS \\
\hline Inferoposterior & $6.2(1.8)$ & $6.6(2.0)$ & NS \\
\hline Septal & $5.3(1.2)$ & $5.9(1.8)$ & NS \\
\hline Lateral & $6.7(2.0)$ & $6.3(1.8)$ & NS \\
\hline Mean & $6.2(1.8)$ & $6.3(1.9)$ & NS \\
\hline \multicolumn{4}{|c|}{ Systolic velocity at low dose dobutamine $(\mathrm{cm} / \mathrm{s})$} \\
\hline Anterior & $7.8(3.5)$ & $8.6(2.9)$ & NS \\
\hline Inferoposterior & $8.5(2.5)$ & $8.6(2.9)$ & NS \\
\hline Septal & $6.7(2.0)$ & $7.4(2.4)$ & NS \\
\hline Lateral & $8.2(3.1)$ & $8.8(2.9)$ & NS \\
\hline Mean & $7.8(2.9)$ & $8.3(2.8)$ & NS \\
\hline \multicolumn{4}{|c|}{ Increase in systolic velocity from rest to low dose $(\mathrm{cm} / \mathrm{s})$} \\
\hline Anterior & $1.1(2.0)$ & $2.4(2.6)$ & NS \\
\hline Inferoposterior & $2.2(1.7)$ & $1.9(1.6)$ & NS \\
\hline Septal & $1.4(1.7)$ & $1.5(1.4)$ & NS \\
\hline Lateral & $1.5(1.7)$ & $2.4(2.2)$ & NS \\
\hline Mean & $1.6(2.2)$ & $2.0(2.0)$ & NS \\
\hline
\end{tabular}

Data are presented as mean (SD).

NS, not significant. 
Q waves. The present data show that Q waves do not necessarily represent a transmural infarction; residual viable tissue was present in a high proportion of dysfunctional regions with Q waves on surface ECG.

Quantitative information on regional LV function and contractile reserve in Q wave and non-Q wave dysfunctional regions is lacking. Therefore, this study quantified regional LV function at rest and contractile reserve during infusion of low dose dobutamine in Q wave and non-Q wave dysfunctional regions. In accordance with the previous studies that assessed myocardial viability in Q wave regions, we found viable tissue in $60 \%$ of $\mathrm{Q}$ wave related regions when an increase in Vs of $\geqslant 1 \mathrm{~cm} / \mathrm{s}$ at low dose dobutamine challenge was used to define viability. Dysfunctional non-Q wave regions were more often viable $(73 \%)$, although the difference did not reach significance.

\section{Assessment of viability by TDI}

Dobutamine stress echocardiography can be used to assess the presence of viable myocardium by showing an improvement in myocardial wall motion during low dose dobutamine challenge. ${ }^{6}$ The presence of increased wall thickening at low dose dobutamine infusion with subsequent worsening during higher rates of infusion (biphasic response) has been found to increase the test's sensitivity for the detection of viability. ${ }^{15}$ However, the assessment of myocardial wall motion with dobutamine infusion is subjective, showing low sensitivity to detect minor alterations in myocardial wall motion.

Pulsed wave TDI has the ability to quantify myocardial wall motion velocities. ${ }^{16}{ }^{17}$ Several factors can influence TDI measurements, such as the translational and rotational motion of the heart within the thorax, as well as the angle of incidence of the ultrasound beam relative to the axis of myocardial longitudinal movement. By assessing myocardial velocities from the apical views, the effect of translation and rotation of the heart on the measurement of myocardial velocities is minimised. In addition, the apex acts as a fixed reference point facilitating the assessment of contraction and relaxation in the axial plain without the need for angle correction. ${ }^{18}$ A possible limitation in the evaluation of TDI results may be tethering between adjacent regions that can influence its ability to localise differences in myocardial velocity. To avoid this effect, tissue Doppler tracings were obtained with the sample positioned near the mitral annulus to assess the vectorial sum of contraction velocities of the longitudinally oriented myocardial fibres between the base and the apex. ${ }^{19}$

Pulsed wave TDI is a feasible and relatively inexpensive technique that can increase the sensitivity of the dobutamine stress test, having at the same time a specificity that is comparable with that of the dobutamine stress test. ${ }^{112021}$ Hence, pulsed wave TDI can be used in clinical practice to provide additional information on viability, especially in regions where visual delineation of endocardium by two dimensional echocardiography is suboptimal or interpretation of wall motion is difficult.

\section{Study limitations}

In the current study, TDI was used to assess regional LV function at rest and during low dose dobutamine infusion. However, the results of functional outcome after revascularisation were not examined. Secondly, although the left ventricle was divided into four major regions to compare ECG with echocardiographic data, misalignment may have influenced the study results. ${ }^{22}{ }^{23}$ Lastly, patients who underwent previous revascularisation were included in the study. Myocardial revascularisation might have influenced the presence and the number of Q waves, as well as the presence of viable myocardium. Nevertheless, the time interval between revascularisation and the current study was more than a year in all patients.

\section{Conclusions}

Quantitative TDI showed comparable wall motion velocities at rest and during low dose dobutamine infusion in dysfunctional regions with and without Q waves. Among dysfunctional regions, Q waves on the ECG do not indicate more severe dysfunction and myocardial contractile reserve was comparable in $\mathrm{Q}$ wave and non-Q wave dysfunctional myocardium. Hence, in patients with LV dysfunction caused by chronic coronary artery disease, viability should be assessed non-invasively irrespective of the presence of $\mathrm{Q}$ waves on the surface ECG.

\section{Authors' affiliations}

M Bountioukos, A F L Schinkel, V Rizzello, R Rambaldi, E C Vourvouri, J R T C Roelandt, D Poldermans, Thoraxcentre, Department of Cardiology, Erasmus Medical Centre, Rotterdam, Netherlands J J Bax, Department of Cardiology, Leiden University Medical Centre, Leiden, Netherlands

\section{REFERENCES}

1 Antaloczy Z, Barcsak J, Magyar E. Correlation of electrocardiologic and pathologic findings in 100 cases of $Q$ wave and non- $Q$ wave myocardial infarction. J Electrocardiol 1988;21:331-5.

2 Sullivan W, Vlodaver Z, Tuna N, et al. Correlation of electrocardiographic and pathologic findings in healed myocardial infarction. Am J Cardiol 1978;42:724-32

3 Tillisch J, Brunken R, Marshall R, et al. Reversibility of cardiac wall-motion abnormalities predicted by positron tomography. N Engl J Med 1986;314:884-8

4 Brunken R, Tillisch J, Schwaiger M, et al. Regional perfusion, glucose metabolism, and wall motion in patients with chronic electrocardiographic $Q$ wave infarctions: evidence for persistence of viable tissue in some infarct regions by positron emission tomography. Circulation 1986;73:951-63.

5 Hashimoto T, Kambara H, Fudo T, et al. Non- $Q$ wave versus $Q$ wave myocardial infarction: regional myocardial metabolism and blood flow assessed by positron emission tomography. J Am Coll Cardiol 1988;12:88-93.

6 Bax JJ, Poldermans D, Elhendy A, et al. Improvement of left ventricular ejection fraction, heart failure symptoms and prognosis after revascularization in patients with chronic coronary artery disease and viable myocardium detected by dobutamine stress echocardiography. J Am Coll Cardiol 1999;34:163-9.

7 Lee KS, Marwick TH, Cook SA, et al. Prognosis of patients with left ventricular dysfunction, with and without viable myocardium after myocardial infarction. Relative efficacy of medical therapy and revascularization. Circulation 1994; $90: 2687-94$

8 Eitzman D, al-Aouar Z, Kanter HL, et al. Clinical outcome of patients with advanced coronary artery disease after viability studies with positron emission tomography. J Am Coll Cardiol 1992;20:559-65.

9 Anderson WD, Wagner NB, Lee KL, et al. Evaluation of a QRS scoring system for estimating myocardial infarct size. VI: identification of screening criteria for non-acute myocardial infarcts. Am J Cardiol 1988;61:729-33.

10 Sevilla DC, Wagner NB, Anderson WD, et al. Sensitivity of a set of myocardial infarction screening criteria in patients with anatomically documented single and multiple infarcts. Am J Cardiol 1990;66:792-5.

11 Rambaldi R, Poldermans D, Bax JJ, et al. Doppler tissue velocity sampling improves diagnostic accuracy during dobutamine stress echocardiography for the assessment of viable myocardium in patients with severe left ventricular dysfunction. Eur Heart J 2000;21:1091-8.

12 Bax JJ, Cornel JH, Visser FC, et al. Prediction of improvement of contractile function in patients with ischemic ventricular dysfunction after revascularization by fluorine-18 fluorodeoxyglucose single-photon emission computed tomography. J Am Coll Cardiol 1997;30:377-83.

13 Sciagra R, Bisi G, Santoro GM, et al. Comparison of baseline-nitrate technetium-99m sestamibi with rest-redistribution thallium-201 tomography in detecting viable hibernating myocardium and predicting postrevascularization recovery. J Am Coll Cardiol 1997;30:384-91.

14 Senior R, Kaul S, Raval U, et al. Impact of revascularization and myocardial viability determined by nitrate-enhanced Tc-99m sestamibi and Tl-201 imaging on mortality and functional outcome in ischemic cardiomyopathy. J Nud Cardiol 2002;9:454-62.

15 Cornel JH, Bax JJ, Elhendy A, et al. Biphasic response to dobutamine predicts improvement of global left ventricular function after surgical revascularization in patients with stable coronary artery disease: implications of time course of recovery on diagnostic accuracy. J Am Coll Cardiol 1998;31:1002-10.

16 Yamada E, Garcia M, Thomas JD, et al. Myocardial Doppler velocity imaging: a quantitative technique for interpretation of dobutamine echocardiography. Am J Cardiol 1998;82:806-9. 
17 Katz WE, Gulati VK, Mahler CM, et al. Quantitative evaluation of the segmental left ventricular response to dobutamine stress by tissue Doppler echocardiography. Am J Cardiol 1997;79:1036-42.

18 Gibson DG, Doran JH, Traill TA, et al. Regional abnormalities of left ventricular wall movement during isovolumic relaxation in patients with ischemic heart disease [abstract]. Eur J Cardiol 1978;7(suppl):251-64.

19 Price DJ, Wallbridge DR, Stewart MJ. Tissue Doppler imaging: current and potential clinical applications [abstract]. Heart 2000;84(suppl 2):II1 1-8.

20 Altinmakas S, Dagdeviren B, Uyan C, et al. Prediction of viability by pulsedwave Doppler tissue sampling of asynergic myocardium during low-dose dobutamine challenge. Int J Cardiol 2000;74:107-13.
21 Larrazet F, Pellerin D, Prigent A, et al. Quantitative analysis of hibernating myocardium by dobutamine tissue Doppler echocardiography. Am J Cardio $2001 ; 88: 418-22$

22 Giannuzzi P, Giordano A, Imparato A, et al. Reliability of standard electrocardiogram in detecting left ventricular asynergy in 315 patients with recent myocardial infarction. Clin Cardio patients with recent

23 Savage RM, Wagner GS, Ideker RE, et al. Correlation of postmortem anatomic findings with electrocardiographic changes in patients with myocardial infarction: retrospective study of patients with typical anterior and posterior infarcts. Circulation 1977;55:279-85.

\section{IMAGES IN CARDIOLOGY}

\section{Spontaneous coronary artery dissection in a pre-menopausal woman presenting with transient ST segment elevation}

\begin{abstract}
A
45 year old, pre-menopausal woman, without any known risk factors for coronary artery disease, presented in the emergency department with acute onset of chest pain. The ECG showed transient ST segment elevation of $3 \mathrm{~mm}$ in leads V3-V6. Although creatine kinase (CK) and CK-MB were not increased, troponin I elevation $(3.1 \mathrm{ng} / \mathrm{ml})$ was observed during hospitalisation in the coronary care unit. Because of the transient appearance of the ST segment elevation, thrombolysis was not administered. Instead the patient received nitrates, $\beta$ blockers, aspirin, and heparin and her symptoms were controlled.

Coronary angiography showed a long segment dissection extending from the proximal left anterior descending artery (LAD) to the distal segment of the vessel (panel A). Contrast media entrapment was observed at the origin of the dissection (panel B). The left ventriculogram showed pronounced hypokinesia of the anterolateral and apical segments with a calculated ejection fraction of $40 \%$.

Because of the transient ST segment elevation, there was a high probability of
\end{abstract}

coronary spasm or spontaneous dissection of the LAD, as the ECG changes were present in the anterior leads. Therefore, a thrombolytic agent was not administered and the conservative approach was preferred

E Tsiamis

$\mathrm{K}$ Toutouzas

C Stefanadis ktoutouz@otenet.gr

(A) In left anterior oblique projection the origin (upper arrow) of the dissection is observed in the proximal segment of left anterior

descending coronary artery (LAD). The flap of the dissection ends at the distal segment of the LAD (lower arrow). The dissection is not flow limiting. The left circumflex artery is free of disease. (B) In anteroposterior projection the dissection is clearly observed. The flap of the dissection extends from the proximal (upper arrow) to the distal (lower arrow) segment of the LAD. (C) Immediately after injection of contrast media entrapment of contrast media (arrow) was observed at the proximal segment of the LAD, just distal to the origin of the flap of the dissection.
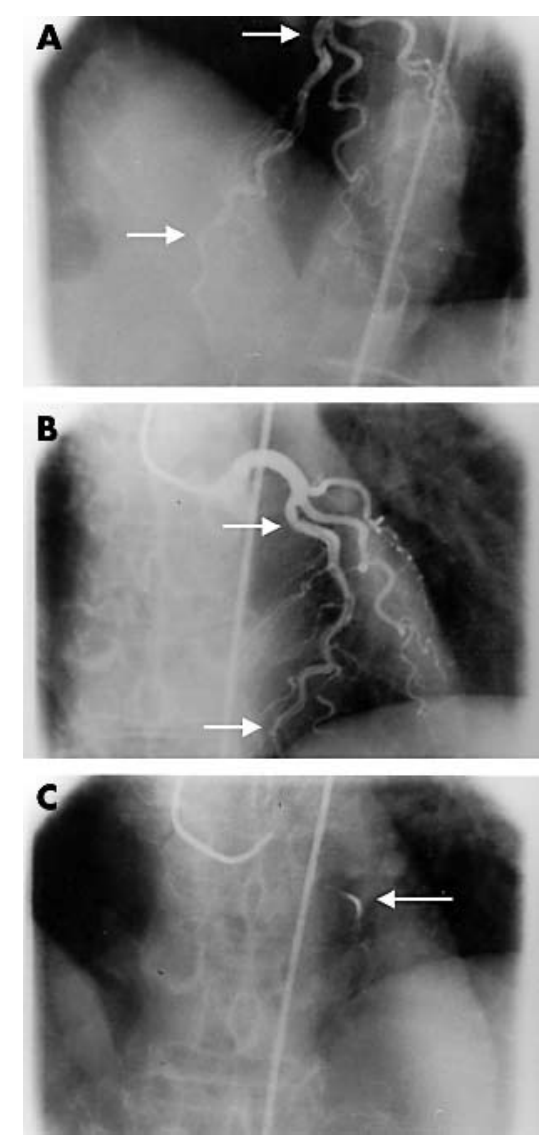